\title{
Der interaktive Perspektivenfilm als Hyperfilm auf Video-DVD
}

\author{
Oliver Jeskulke, Sebastian Hasse, André Melzer, Inga Schön, Michael \\ Herczeg \\ Institut für Multimediale und Interaktive Systeme (IMIS), Universität zu Lübeck
}

\begin{abstract}
Zusammenfassung
Der interaktive Perspektivenfilm (IPF), eine besondere Form des Hyperfilms, unterstützt unter Beibehaltung der bewährten linearen Erzählweise des Mediums Film zu verschiedenen Zeitpunkten des Verlaufs einer Geschichte die aktive Auswahl aus konkurrierenden Erzählperspektiven. Der Zuschauer wird auf diese Weise zum Entscheidungsträger und Gestalter des Informationsflusses. Die bisher weitestgehend auf Navigation reduzierte Interaktivität des Mediums Video-DVD wird somit wesentlich erweitert. Anhand des Filmprojekts „Deine Wahrheit“ wird der Produktionsprozess eines IPF von der Konzeption der Charaktere über das Verfassen des Drehbuchs und der digitalen-technischen Produktion und Nachbearbeitung des Filmmaterials sowie dem abschließenden DVD-Authoring vorgestellt. Belege für den Mehrwert des IPF resultieren aus dem empirischen Vergleich mit einer herkömmlichen, perspektivisch-linearen Version des Films. Das Potenzial dieses neuen Genres wird diskutiert.
\end{abstract}

\section{$1 \quad$ Einleitung}

Menschen haben ein natürliches Interesse an der Erweiterung ihrer Fähigkeiten und Kenntnisse. Dieses Bedürfnis ist nicht nur auf den Erwerb spezieller Fertigkeiten, sondern allgemein auf die Ve rmehrung von Wissen ausgerichtet (z.B. Puca \& Langens 2002). Neues stellt dabei einen wichtigen Anreiz für dieses Neugiermotiv dar: Wir sind grundsätzlich daran interessiert, Sachverhalte aus ,neuen Blickwinkeln“ zu betrachten, neue Informationen aktiv zu suchen und so die Handlungsweisen Anderer zu verstehen. Solche Motive werden auch beim Experience Design (Shedroff 1995; 1997), einer Erweiterung des Interaktionsdesigns adressiert. Dabei werden durch spezielle Motivationskonzepte, insbesondere erlebnisorientierte, interaktive narrative Konzepte (Murray 1997), das Interesse und die Aufmerksamkeit der Benutzer gewonnen und gebunden.

„Citizen Kane“, 1940 von Orson Welles erschaffen, stellt in diesem Zusammenhang nicht zuletzt deshalb ein Meisterwerk des Kinos dar, weil der Film das evolutionsbiologisch ver- 
ankerte Neugiermotiv aus motivationspsychologischer Sicht erfolgreich bedient. Welles' Konzept bestand in der zu seiner Zeit revolutionären Erzählweise, die Geschichte in Rückblenden zu erzählen, den Hauptcharakter dabei aus verschiedenen subjektiven Sichtweisen zu beleuchten und die Bewertung der Ereignisse letztlich dem Urteil des Zuschauers zu überlassen. Allerdings folgt „Citizen Kane“ - wie zahlreiche Filme danach - insofern traditionellen Strukturen, als dass die verschiedenen Perspektiven zeitlich nacheinander und für den Zuschauer ohne Interaktionsmöglichkeit präsentiert we rden.

Der von uns entwickelte experimentelle interaktive Perspektivenfilm (IPF) „Deine Wahrheit" greift die Idee unterschiedlicher Perspektiven im Film auf: Vor jedem Kapitel der Story hat der Betrachter über Interaktionselemente in der Menüsteuerung die Möglichkeit, eine von fünf Erzählperspektiven auszuwählen. Auf der Basis bestehender Ansätze wurde das Hypermedium Video-DVD somit in seiner Struktur und Funktionalität dahingehend erweitert, dass eine aktive Wahl zwischen den in zeitlicher Konkurrenz angebotenen Ezählperspektiven stattfindet, ohne dass der lineare Charakter eines Spielfilms verloren geht. Der IPF führt damit die Idee von Hypermedia (Woodhead 1991) und Hyperfiction (Sassòn-Henry 1999; Zellweger et al. 2002) in Form des Hyperfilms fort. Das Grundprinzip wurde bereits in den verschiedenen Formen des interaktiven Fernsehens aufgegriffen, scheiterte dort bislang jedoch insbesondere am fehlenden oder unzulänglichen Rückkanal sowie an der Realisierung geeigneter Infrastrukturen für Video-on-Demand (VoD). Durch die allgemeine Verfügbarkeit der DVD entstehen neue Verbreitungs- und Nutzungsmöglichkeiten des Hyperfilms. Andere Konzepte zum Hyperfilm beschreibt u.a. Tua (2002). Es kann erwartet werden, dass der Hyperfilm auf DVD neben interaktiven Spielen einen besonderen Stellenwert unter künftigen interaktiven Medien im Consumer-Bereich erreichen kann. Bislang gibt es allerdings wenige kontrollierte Untersuchungen, die die besondere motivatorische Wirkung des Hyperfilms untersucht haben. Hier versucht die vorliegende Studie einen Beitrag zu leisten.

\section{Medien und Interaktivität}

Mit dem Begriff der Informationsgesellschaft wird das gesellschaftliche Phänomen der umfangreichen Nutzung von informationstechnologischen Mitteln beschrieben (z.B. Halbach \& Faßler 1998). Dabei ist zu beobachten, dass sich die traditionellen Rollen von Konsument und Produzent zunehmend auflösen; der bisherige Konsument nimmt zunehmend aktiv Einfluss auf das Geschehen des ihm Dargebotenen (so schlägt etwa Toffler (1980) den Begriff des Prosumenten vor). Prominentestes Beispiel dieser Entwicklung ist das WorldWideWeb (WWW), das als das erste interaktive Massenmedium angesehen werden kann. Das WWW erlaubt bei der Lektüre von Hypertextdokumenten, interessant erscheinenden Querverweisen mit einemeinfachen Knopfdruck über den gesamten Globus zu folgen. Aufgrund der offenen Struktur des WWW kann prinzipiell jeder Teilnehmer seine individuellen Fähigkeiten und Kenntnisse, Meinungen und Interessen in das Medium einfließen lassen. Tatsächlich haben inzwischen Millionen von Nutzern Informationsangebote für das WWW aufbereitet und der Öffentlichkeit zur Verfügung gestellt. 
Ein relativ neues Medium ist die Video-DVD. Als digitaler Massen-, insbesondere Videospeicher für Aufnahmen in höchster Qualität konzipiert, bietet sie erhebliches Interaktionspotenzial. Allerdings ist die Interaktivität der meisten DVDs lediglich als Navigation in Bezug auf die verschiedenen Inhalte implementiert (z.B. Wahl einzelner Kapitel) und damit auf eine Funktion beschränkt, über die prinzipiell bereits die Video-Kassette verfügte. Dabei eignet sich die DVD unter anderem deshalb besonders als Trägermedium, da sie eine Navigation mit Hilfe interaktiver Menüs erlaubt. Diese Menüs werden dabei technisch als sogen. Subpicture-Overlays realisiert, die an jeder Stelle auf der DVD über das normale Bild gelegt we rden können. Im Projekt „Deine Wahrheit“ wurde geprüft, inwiefern eine Erweiterung der Struktur der DVD als Hypermedium (Hyperfilm) durch Implementierung einer zusätzlichen Interaktionsebene - die Wahl der Erzählperspektive als Menüoption - zu einer positiv bewerteten, neuen Form der multimedialen Erfahrung führt. Diese Fragestellung wurde am Be ispiel des populären Mediums Film untersucht.

\section{$3 \quad$ Produktion des interaktiven Perspektivenfilms}

Der Wandel alter Medien in neue infolge der voranschreitenden Entwicklung digitaler Technologien wurde bereits früh mit dem Wunsch nach zusätzlichen, interaktiven Elementen verbunden. In Bezug auf das Medium Film äußerte Youngblood bereits 1970 (zit. n. Gansing 2003) die Hoffnung, das Kino um entsprechende Möglichkeiten zu erweitern. Mittlerweile liegen diesbezüglich zahlreiche Beispiele vor, wie etwa die Ausstellung Future Cinema des Zentrums für Kunst und Medientechnologie (ZKM) in Karlsruhe dokumentierte (URL: http://www.zkm.de/futurecinema, v. 02.03.2004).

Die individuelle Perspektivenwahl wird eindrucksvoll in der an den Film Twelve Angry Men (USA, 1957; deutscher Titel Die zwölf Geschworenen) angelehnten Installation Three Angry Men (MacIntyre et al. 2002) demonstriert. Diese Installation bietet verschiedene konkurrierende Ebenen, zwischen denen der Zuschauer wechseln kann. Dabei folgen alle Ebenen in Three Angry Men einer gemeinsamen Zeitlinie. In einer Augmented-Reality-Umgebung wird der Benutzer durch die Verknüpfung von realem Raum und virtuellen Charakteren in die Lage versetzt, dem Gespräch der Charaktere zu folgen und gleichzeitig die Gedanken desjenigen Charakters nachzuvollziehen, den er gleichsam „bewohnt“. Mit jedem Wechsel des Charakters gewinnt der Benutzer mehr Information, erweitert damit sein Wissen und erhält somit ein insgesamt besseres Verständnis der unterschiedlichen Charaktere.

\subsection{Anforderungsanalyse}

Für eine erfolgreiche Umsetzung des IPF „Deine Wahrheit“ erbrachte die Planungsanalyse zahlreiche Anforderungen an Bedienung, Filmparameter sowie technische Merkmale. So sollte das filmische Endprodukt dem Benutzer bei minimaler Führung eine möglichst große Entscheidungsfreiheit überlassen, ohne dass dabei sein Wissen über die Größe des möglichen Handlungsraumes verloren gehen durfte. Gleichzeitig sollten Entscheidungen unzulässig 
sein, die den Benutzer in eine Sackgasse führen. Der Film sollte eine hohe externe Validität aufweisen und sich daher bezüglich der Qualität von Bild, Ton und Inhalt einem gängigen Fernsehfilm annähern. Zudem war darauf zu achten, dass die Interaktion sinnstiftend blieb, dem Film also ein echter Mehrwert hinzugefügt wurde. Darüber hinaus sollten Redundanzen vermieden werden, so dass der Film auch bei erneuter Betrachtung als genauso interessant empfunden wird. Besonderes Ziel war es, die Interaktionspunkte im Film so zu implementieren, dass ein möglichst direkter Transport der Inhalte des Films zum Benutzer gesichert war. Alle Ablenkungen vom Inhalt, etwa dadurch, dass das Trägermedium als solches wahrnehmbar wird, sollten vermieden werden. Im IPF „Deine Wahrheit" wurden daher die Interaktionspunkte als wiederkehrendes Motiv (Verlassen einer Wohnung) in die Handlung integriert. Der Zuschauer greift jeweils zu einem Zeitpunkt über ein Auswahlmenü in den Film aktiv ein, der den „natürlichen“ Abschluss eines Erzählkapitels und den Übergang in einen neuen Filmabschnitt darstellt. Die Bedeutung sorgfältiger Konzeption des Designs von Interaktionselementen im Film stellt Gansing (2003) heraus:

“(...) we cannot ignore the importance of interaction design as it enters into dialogue with narrative structures. Just as stylistic conventions regulate narrative understanding, interaction design regulates possible actions." (40f.)

\subsection{Konzept}

Grundlage des IPF „Deine Wahrheit“ ist eine einheitliche Geschichte, die unabhängig von den Interaktionen des Benutzers erzählt wird. Durch die Implementierung aktiver Interakt ionsmöglichkeiten soll de natürliche Motivation des Zuschauers, neue Informationen zu sammeln, unterstützt werden. Gleichzeitig ist sicherzustellen, dass die Interaktion ohne Einfluss auf die Geschichte an sich bleibt, oder der lineare Spielfilm-Charakter verloren geht.

Analog uır Wirkungsweise von Kamerafiltern sollen dazu die Erzählperspektiven unterschiedlicher Filmcharaktere die Sicht des Zuschauers auf die erzählte Geschichte „filtern“. Den theoretischen Hintergrund dieser Überlegung bilden kognitionspsychologische Überlegungen zur Subjektivität menschlicher Wahrnehmungsvorgänge. Individuelle Wahrnehmu ngen sind demnach das Ergebnis komplexer Wechselwirkungen von eingehenden Daten mit den Erfahrungen, Einstellungen und Erwartungen des Wahrnehmenden (für eine Übersicht siehe z.B. Goldstein 2002). Daraus resultiert, dass subjektiv als ,wahr“ Empfundenes in dem Maße zu ergänzen bzw. zu relativieren ist, wie konkurrierende Wahrnehmungen verfügbar werden. Die im IPF „Deine Wahrheit“ realisierte Interaktionsmöglichkeit dient daher insofern als Filter, als dass der Zuschauer durch den Wechsel zwischen unterschiedlichen Perspektiven lediglich verschiedene Wahrnehmungen derselben Geschichte erhält. Deren Reflexion soll insgesamt eine veränderte Rezeption der filmischen Ereignisse induzieren. Der Zuschauer wird in „Deine Wahrheit“ als Entscheidungsträger miteinbezogen, er soll letztlich erkennen, dass er selber für sein Bild von den Geschehnissen verantwortlich ist.

Die Geschichte des IPF „Deine Wahrheit“ wird in fünf Kapiteln erzählt. Vor jedem Kapitel besteht per Menüauswahl die Möglichkeit eine von fünf Erzählperspektiven für das nachfolgende Kapitel auszuwählen. Insgesamt kann der Film somit auf fünf hoch fünf (3125) ver- 
schiedene Arten gesehen werden. Der gesamte Film kann auch in einer (durchgängigen) Perspektive ohne Berücksichtigung der anderen vier Perspektiven gesehen werden. Abbildung 1 illustriert die Stru ktur des IPF.

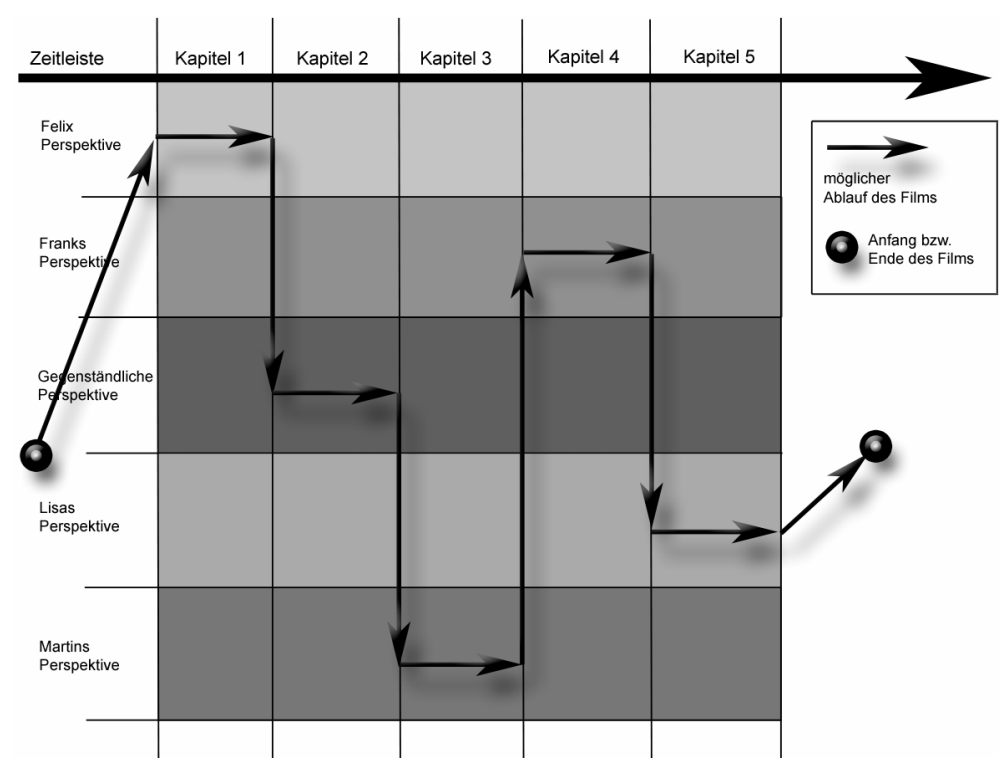

Abbildung 1: Struktur des IPF „Deine Wahrheit“ mit möglichem Betrachtungsverlauf.

\subsection{Geschichte, Charaktere der Handlung und Drehbuch}

„Deine Wahrheit“ stellt eine Variante einer Kriminalgeschichte dar, in der es in Rückblenden um den verschwundenen Hauptcharakter Tracy geht. Der Zuschauer begleitet Tracys Bruder auf der Suche nach den Gründen für das Verschwinden. Entscheidend ist, dass der Zuschauer bestimmt, welche Person der Bruder aus Tracys Umfeld trifft und in welcher Erzählperspektive das nachfolgende Kapitel präsentiert wird. Am Ende eines Kapitels verlässt der Zuschauer mit dem Bruder die jeweils besuchte Wohnung und wählt über das interaktive Menü die Perspektive des nächsten Kapitels. Dazu nimmt die Kamera im Film die Perspektive des Bruders ein, und der Zuschauer trifft mithilfe der Fernbedienung eine Auswahl zwischen den Alternativen in dessen Notizbuch (Abbildung 2). 


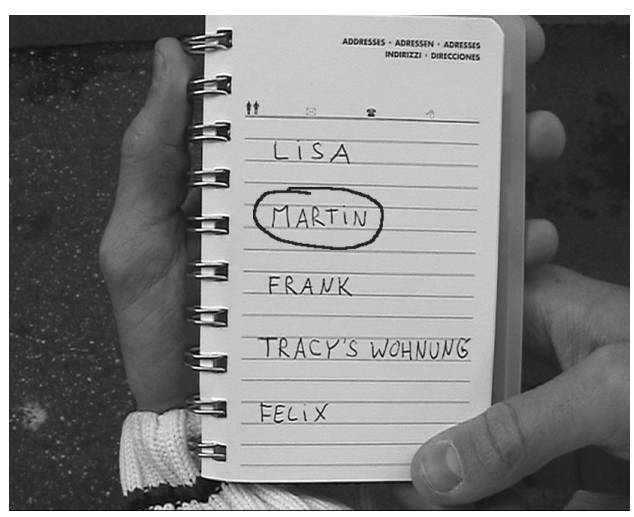

Abbildung 2: Auswahlmenü an den Interaktionspunkten im IPF „Deine Wahrheit“.

Aus den genannten konzeptuellen Überlegungen wurde bei Konzeption und Gestaltung von Geschichte und Drehbuch des IPF besonderes Gewicht auf Authentizität der Dialoge und Charakterzeichnung gelegt. Um den als Mittelpunkt der Geschichte konzipierten Hauptcharakter Tracy herum gruppieren sich die Charaktere seiner Freunde Felix und Martin, seines Gegenspielers Frank und seiner Freundin Lisa. In jeder der fünf Perspektiven (die vier Charaktere sowie die gegenständliche Perspektive „Tracys Wohnung“) werden bestimmte Charaktereigenschaften von Tracy besonders hervorgehoben (Abbildung 3). Diese Vorgaben wurden anschließend im Drehbuch mit der jeweiligen Erzählsituation in Szenen und Dialoge umgesetzt.

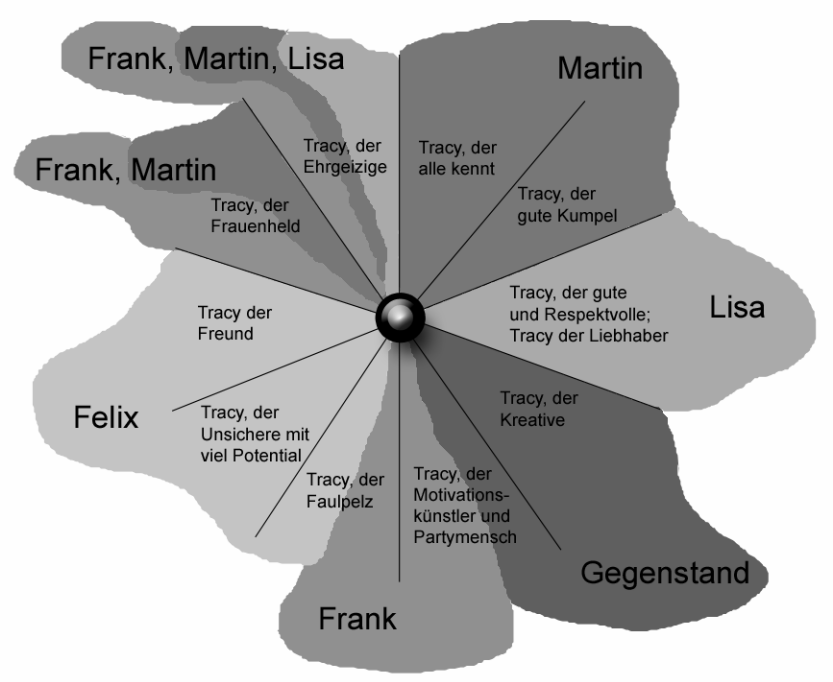




\subsection{Dreharbeiten, Nachbearbeitung, DVD-Authoring}

Die Komplexität des Projektes erforderte die Entwicklung einer Datenbank gestützten Software zur Drehplanung. Auf diese Weise konnten die Rollen und Schauspieler, deren Verfügbarkeit sowie die mehr als 200 Szenen effizient verwaltet werden. Gedreht wurde mit Sony Mini-Digitalkameras. Der Ton wurde mit externem Mikro auf DAT-Band aufgezeichnet.

Die insgesamt 25 gedrehten Film-Episoden (eine Episode ist ein Kapitel in einer Erzählperspektive) variieren in ihrer Länge zwischen acht und 13 Minuten. Nach Abschluss der Schneide- und Nachbearbeitungsaufgaben wurden die Filmteile exportiert und in MPEG-IIFormat u mgewandelt.

Der Film wurde auf zwei DVDs verteilt (DVD-5, durchschnittl. Datenrate: $3.5 \mathrm{mbit} / \mathrm{s}$ ). Zwar wird der Zuschauer dadurch gezwungen, die DVD zu wechseln und sich somit auf die Benutzerebene zurück zu begeben, doch hätte ein Verkürzen der Filmlänge bzw. eine Verminderung der Bild- und Tonqualität eine signifikantere Störung in der Rezeption des Films dargestellt. Für das Storyboard wurde festgelegt, dass die Zwischenmenüs für den Zuschauer nach Möglichkeit nicht direkt als Interaktion bewusst werden sollten. Sie wurden daher in die Handlung integriert, so dass sie bildlich genau an das vorangegangene Video anschließen. Nach Auswahl einer Erzählperspektive für das nächste Kapitel läuft der Film an der Stelle weiter, wo er durch das Menü unterbrochen wurde (s. Abbildung 4).

\section{$4 \quad$ Empirische Prüfung des IPF}

Im Anschluss an die Produktion des IPF „Deine Wahrheit“ wurden insgesamt 28 Personen eingeladen, den IPF unter Testbedingungen zu schauen. In Einzeltestung wurde die DVD an einem PC oder Notebook präsentiert. Jede Person sah den vollständigen Film genau dreimal. Zuvor waren die Personen zufällig einer von zwei Gruppen zugeordnet worden. Die Mitglieder der einen Gruppe (nachfolgend ,interaktive Bedingung ") wurden ohne weitere Vorgaben angewiesen, die Erzählperspektive an den Interaktionspunkten vor den Kapiteln mö glichst oft zu wechseln. In der zweiten Gruppe (nachfolgend „lineare Bedingung“) war eine feste Abfolge der drei Durchgänge in jeweils einer durchgängigen Perspektive vorgegeben. Diese Gruppe sah den IPF somit wie einen herkömmlichen Perspektivenfilm in sukzessiver Abfolge der verschiedenen Perspektiven.

Im Anschluss an den dritten Filmdurchgang bearbeiteten alle Testpersonen einen 22 Items umfassenden Fragebogen ${ }^{1}$, der zugleich den Abschluss der Untersuchung darstellte.

\footnotetext{
${ }^{1}$ In der interaktiven Bedingung waren zusätzlich sechs Items zur Navigation und Interaktivität zu beantworten.
} 


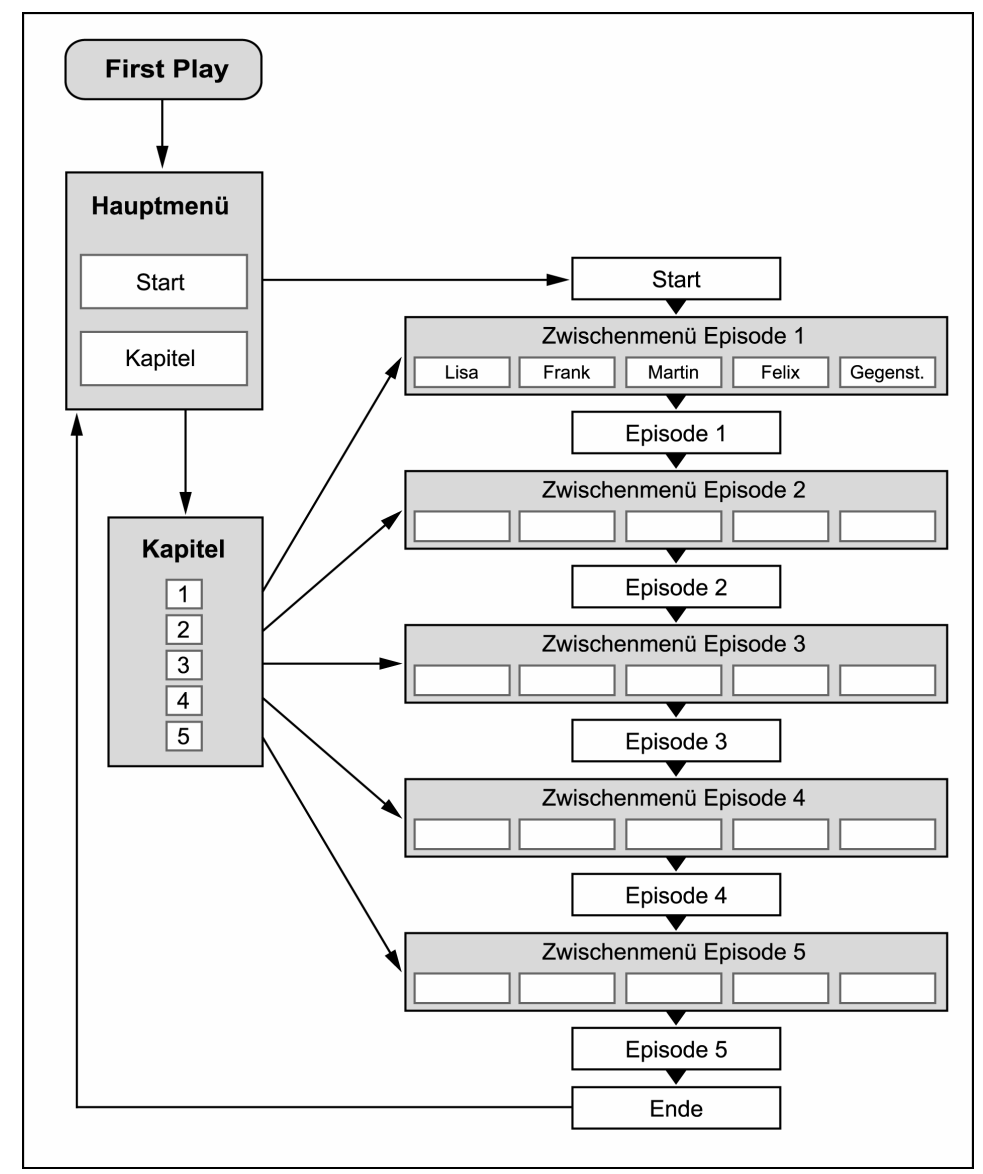

Abbildung 4: Das Storyboard im IPF „Deine Wahrheit“.

\subsection{Ergebnisse}

Soweit nicht anders vermerkt, erfolgte ein Urteil jeweils durch Ankreuzen auf einer vierstufigen Skala. Die Antworten wurden mit den Werten 1 (,Ich stimme überhaupt nicht zu“), 2 (,Ich stimme kaum zu“), 3 (,,Ich stimme zu“) bzw. 4 (,Ich stimme voll zu“) kodiert. Nachfolgende Angaben beziehen sich jeweils auf das mittlere Gruppenurteil.

Die Analyse des Abschlussfragebogens erbrachte statistisch bedeutsame Gruppenunterschiede (jeweils $t$-Tests für unabhängige Stichproben, df =26, a = .05). Obwohl der Film durchweg positiv beurteilt wurde, bewerteten die Teilnehmer der interaktiven Bedingung $(M=$ 3.71) zum Abschluss der Untersuchung den Kapitel weisen Wechsel der Perspektive signifikant positiver als die Teilnehmer der linearen Bedingung dies für die herkömmliche Betrachtungsweise taten $(M=3.07 ; t=3.11)$. Die gelungene Umsetzung des im IPF ,Deine Wahr- 
heit “ gewählten Navigationskonzepts der Interaktion (in die Filmhandlung integrierte Wahl der Erzählperspektive über das Notizbuch, s. Abbildung 2) ist darüber hinaus durch die sehr hohe Zustimmung in der interaktiven Bedingung $(M=3.79)$ belegt; hier wurde zudem weder das Vorhandensein $\mathrm{zu}$ vieler $(M=1.43)$, noch $\mathrm{zu}$ weniger $(M=2.07)$ Interaktionspunkte kritisiert. Offenbar gelang mit dem IPF auch eine bessere Vermittlung der einzelnen Chara ktere der Handlung: Der Aussage, sich den Charakteren so wie in bevorzugten Fernsehserien vertraut zu fühlen, stimmten die Teilnehmer der interaktiven Bedingung $(M=3.07)$ vergleichsweise stärker zu (lineare Bedingung: $M=2.50 ; t=2.66$ ).

In Bezug auf die allgemeine Beurteilung des Perspektivenfilms wurde die Möglichkeit, Sachverhalte aus unterschiedlichen Perspektiven betrachten zu können, trotz der damit einhergehenden Wiederholung von Ereignissen generell begrüßt. Hier fiel die Zustimmung der Teilnehmer in der linearen Bedingung $(M=3.64)$ sogar substanziell höher aus, als in der interaktiven Bedingung $(M=3.21 ; t=2.10)$. Zudem war in der linearen Bedingung $(M=$ 3.50) ein tendenziell stärker ausgeprägtes Bedauern zu verzeichnen (interaktive Bedingung: $M=3.00 ; t=1.84, \mathrm{p}=.08)$, nicht sämtliche Perspektiven bzw. Episoden sehen zu können.

\subsection{Diskussion}

Für den direkten Vergleich des IPF mit dem traditionell-linearen Perspektivenfilm bleibt festzuhalten, dass die Zuschauer die zusätzlichen Möglichkeiten der interaktiven Einflussnahme positiver beurteilten. Sie fühlten sich zudem in Bezug auf Navigation und Nutzung weder überfordert noch verwirrt. Darüber hinaus sind sie vergleichsweise stärker davon überzeugt, alle Facetten des Charakters einer handelnden Person kennen gelernt zu haben. Wie erwartet, besteht der Vorteil des IPF somit darin, das ohnehin ausgeprägte Bedürfnis zur Perspektivenübernahme durch zusätzliche Steuerungsmöglichkeiten substanziell zu fö rdern.

\section{$5 \quad$ Fazit}

Der hier am Beispiel „Deine Wahrheit“ vorgestellte IPF greift gegenwärtige Entwicklungen in der Medienlandschaft auf und gibt sie an den Zuschauer weiter. Ohne die bewährte lineare Erzählweise des Mediums Film zu verlassen, erweitert der IPF mit der Video-DVD als Hypermedium die Idee der Hyperfiction (Sassòn-Henry 1999; Zellweger et al. 2002) und verbindet diese mit bestehenden erlebnisorientierten, interaktiven narrativen Konzepten (Murray 1997) und Konzepten des Experience Designs (Shedroff 1995; 1997). Mit Hilfe behutsam in die Filmhandlung implementierter Interaktionsschnittstellen wird der Zuschauer aktiv an der Unterhaltung beteiligt. Der IPF folgt somit einer Konzeption, die Interaktivität in den digitalen Medien als Externalisierung mentaler Prozesse definiert (Manovich 2001). Es wurde gezeigt, dass der Mehrwert des IPF unter anderem in der erfolgreichen Förderung der natürlichen Motivation des Zuschauers besteht, seine Fähigkeiten und Kenntnisse zu erweitern und ein verstärktes Verständnis für andere Perspektiven zu entwickeln. Insgesamt ist davon 
auszugehen, dass der DVD-basierte Hyperfilm neben anderen Formen künftiger interaktiver Medien eine erfolgreiche Alternative im Consumer-Bereich darstellen kann.

\section{Literaturverzeichnis}

Gansing, K. (2003): The Myth of Interactivity or The Interactivity Myth? Interactive Film as an Imaginary Genre. In: Proceedings of the Fifth International Digital Arts and Culture Conference. RMIT, Melbourne, Australia. May 2003. verfügbar unter http://hypertext.rmit.edu.au/dac/papers/Gansing.pdf, v. 02.03.2004. [S. 39-45].

Goldstein, E. B. (2002): Wahrnehmungspsychologie. Heidelberg: Spektrum Akademischer Verlag.

Halbach, W. R.; Faßler, M. (1998): Einleitung in die Mediengeschichte. In: Faßler, M.; Halbach, W. R. (Hrsg.): Geschichte der Medien. München: Fink. [S. 17-53].

MacIntyre, B.; Bolter, J.; Vaughan, J.; Hannigan, B.; Moreno, E.; Haas, M.; Gandy, M. (2002): Three Angry Men: Dramatizing Point-of-View using Augmented Reality. In: SIGGRAPH 2002 Technical Sketches. San Antonio, TX, July 2002. [S. 268].

Manovich, L. (2001): The Language of New Media. Cambridge, MA: MIT Press.

Murray, J. H. (1997): Hamlet on The Holodeck: The Future of Narrative in Cyberspace. New York: Free Press.

Puca, R. M..; Langens, T. A. (2002): Motivation. In: Müsseler, J.; Prinz, W. (Hrsg.): Allgemeine Psychologie. Heidelberg: Spektrum Akademischer Verlag. [S. 225-269].

Sassòn-Henry, P. (1999): Jorge Luis Borges: A Forerunner of the Technology of the New Millennium. In: ACM Hypertext 1999. Doctoral Consortium Final Report, Aalborg University Technical Report AUE-CS-99-03, verfügbar unter http://www.daimi.au.dk/ pnuern/ht99dc/sasson/final.pdf , v. 02.03.2004.

Shedroff, N. (1995): Experience Design. Indianapolis, IN: New Riders Publishing.

Shedroff, N. (1999): Information Interaction Design: A Unified Field Theory of Design. In Jacobsen, R. (Hrsg.): Information Design. Cambridge: MIT Press. [S. 267-292].

Toffler, A. (1980): Die Zukunftschance: Von der Industriegesellschaft zu einer humaneren Zivilisation, München: Bertelsmann.

Tua, R. (2002): From Hyper-Film to Hyper-Web: Continuation of a European Project. In: Cappellini, V.; Hemsley, J.; Stanke, G. (Hrsg.): EVA 2002 Proceedings, Bologna.

Woodhead, N. (1991): Hypertext and Hypermedia: Theory and Applications. Boston, MA: AddisonWesley Longman.

Zellweger, P. T.; Mangen, A.; Newman, P. (2002): Narratives and Literary Hypertext: Reading and Writing Fluid Hypertext Narratives. In: Proceedings of the thirteenth ACM conference on Hypertext and Hypermedia. College Park, Maryland, USA. June 11-15, 2002. [S. 45-54].

\section{Danksagung}

Die Autoren danken Frau Sonja Arend vom IMIS (Universität zu Lübeck) für die Unterstützung bei der Erstellung von Abbildung 4. 


\section{Kontaktinformationen}

Dr. André Melzer

Institut für Multimediale und Interaktive Systeme (IMIS)

Universität zu Lübeck

MediaDocks

Willy-Brandt-Allee 31a

D-23554 Lübeck

Email: melzer@imis.uni-luebeck.de

Telefon: 0451/2803 4208

Fax: 0451/2803 4190 